\title{
Evolución de síntomas a corto plazo y grado de satisfacción del paciente tras hemorroidectomía convencional
}

\author{
Angelo Fulle C. ${ }^{1,2}$, Pablo Moreno L. ${ }^{1}$, Felipe Quezada D. ${ }^{1,2}$, Gonzalo Carvajal G. ${ }^{1}$, \\ Pamela Briones N. ${ }^{1}$, Rodrigo Kusanovich B. ${ }^{1,2}$ y Richard Castillo R. ${ }^{1,2}$
}

\section{Evolution of short-term symptoms and degree of patient satisfaction after conventional hemorrhoidectomy}

Background: Hemorrhoidectomy is the treatment of choice for symptomatic hemorrhoids, reserved for patients with grade III or IV hemorrhoids or no response to conservative treatment. There are several surgical techniques, whose degree of resolution of symptoms and patient satisfaction has varied results. Objectives: To evaluate in the short term the symptomatology and degree of satisfaction of patients undergoing conventional hemorrhoidectomy in Complejo Asistencial Dr. Sótero del Río. Material and Methods: A retrospective telephone survey was applied to patients undergoing conventional hemorrhoidectomy from June 2015 to January 2016. The survey includes questions about symptoms in the pre-operative, at the month and at 6 or more months post-operated. Bleeding, pruritus, pain, prolapsed sensation and incontinence were evaluated. The statistical analysis considered the results according to the 5 symptoms evaluated in the survey and then dichotomized according to clinical relevance. A significant $p$ value $<0.01$ was considered. Results: 43 patients answered the survey, with a median age of 55 years (17-80). The median evaluation was 8 months (6-12). Considering the symptoms: bleeding, pruritus, pain and prolapse, 35 patients $(79 \%)$ had 3 or more preoperative symptoms considered significant. Persistent follow-up ranges from 9 to $28 \%$. In the statistical analysis with dichotomized symptoms, there is a statistically significant decrease at one month and at 6 or more months post-surgery. Conclusion: Conventional hemorrhoidectomy is well evaluated by patients in relation to a significant resolution of hemorrhoidal disease symptoms. Key words: hemorrhoids; hemorrhoidectomy; conventional hemorrhoidectomy.

\section{Resumen}

Introducción: La hemorroidectomía es el tratamiento de elección para las hemorroides sintomáticas, reservado para pacientes con hemorroides grado III o IV o sin respuesta a tratamiento conservador. Existen diversas técnicas quirúrgicas, cuyo grado de resolución de síntomas y satisfacción por parte del paciente posee resultados variados. Objetivos: Evaluar al corto plazo la sintomatología y grado de satisfacción de los pacientes operados de hemorroidectomía convencional en el Complejo Asistencial Dr. Sótero del Río. Materiales y Métodos: Se realizó una encuesta telefónica retrospectiva a pacientes operados de hemorroidectomía convencional durante junio de 2015 a enero de 2016. Se preguntó por síntomas en el preoperatorio, al mes y a los 6 o más meses posoperados. Se evaluó sangrado, prurito, dolor, sensación de prolapso e incontinencia. El análisis estadístico consideró los resultados según los 5 síntomas evaluados y luego dicotomizados según relevancia clínica. Se consideró un valor $\mathrm{p}$ significativo $<0,01$. Resultados: Cuarenta y tres pacientes respondieron la encuesta, con una mediana de edad de 55 años (17-80). La mediana de evaluación fue de 8 meses (6-12). Considerando los síntomas: sangrado, prurito, dolor y prolapso, 35 pacientes $(79 \%)$ presentaban 3 o más síntomas preoperatorios considerados significativos. La persistencia al seguimiento varía entre un 9 a un 28\%. En el análisis estadístico con síntomas dicotomizados, existe una disminución estadísticamente significativa al mes y a los 6 o más meses posoperados. Discusión: La hemorroidectomía convencional es bien evaluada por los pacientes en relación a una resolución significativa de síntomas de la enfermedad hemorroidal.

Palabras clave: hemorroides; hemorroidectomía; hemorroidectomía convencional.

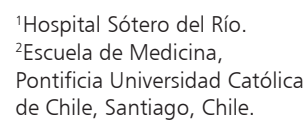

Recibido el 21 de septiembre, aceptado para publicación el 18 de noviembre de 2017

Correspondencia a: Dr. Richard Castillo R. richardcr@gmail.com 


\section{Introducción}

La enfermedad hemorroidal es una de las patologías perianales benignas más frecuentes en la población, representando parte importante de la actividad ambulatoria y quirúrgica coloproctológica ${ }^{1}$. A nivel internacional, se ha estimado que aproximadamente un $5 \%$ de la población general se ve afectada por hemorroides sintomáticas, siendo un $50 \%$ personas mayores de 50 años ${ }^{2-4}$. A nivel nacional, no existen estudios epidemiológicos concluyentes.

El tratamiento de las hemorroides sintomáticas es escalonado, de acuerdo al tipo y grado de hemorroides, síntomas y progresión de la enfermedad, que va desde el tratamiento conservador al quirúrgico. El manejo conservador implica cambios en la dieta y el estilo de vida, asociado o no a fármacos de uso tópico y procedimientos locales como escleroterapia, ligaduras hemorroidales, entre otros ${ }^{1,5}$. La extirpación quirúrgica o pexia son métodos eficaces para el tratamiento de las hemorroides sintomáticas, que se reserva para pacientes con hemorroides grado III o IV, o sin respuesta a tratamiento conservador ${ }^{5-7}$. Dentro de las alternativas quirúrgicas se puede mencionar la hemorroidectomía convencional, considerada el gold estándar de tratamiento quirúrgico ${ }^{8}$.

El síntoma más frecuente es la rectorragia, siendo en general de poca cuantía y que en casos de cronicidad puede ser causa de anemia. El segundo síntoma en frecuencia es el prolapso, seguido de prurito anal e incontinencia fecal de intensidad variable ${ }^{9}$.

La evidencia actual describe una baja recurrencia de síntomas con el uso de la hemorroidectomía convencional, pero se asocia a un importante dolor posoperatorio ${ }^{10,11}$. Desde la perspectiva del paciente, la literatura internacional reporta un alto nivel de satisfacción con el uso de esta técnica en los seguimientos a largo plazo, mediante el uso de preguntas de evaluación directa de satisfacción ${ }^{8,12}$.

A nivel nacional, existe poca evidencia de la percepción del paciente en la evolución de su sintomatología y valoración del tratamiento quirúrgico de la enfermedad hemorroidal.

El objetivo de este trabajo es evaluar a corto plazo, la evolución de los síntomas y grado de satisfacción de pacientes sometidos a hemorroidectomía convencional en la unidad de cirugía ambulatoria del Complejo Asistencial Dr. Sótero del Río (CASR).

\section{Material y Métodos}

En el presente trabajo se incluyó a todos los pacientes operados de hemorroidectomía convencional electiva en el Servicio de Cirugía Ambulatoria del CASR, entre junio de 2015 a enero de 2016. Todos ellos fueron evaluados a los 6 o más meses posoperados con una encuesta telefónica retrospectiva.

Se excluyó a los pacientes con diagnóstico asociado de fisura anal, fístula anorrectal, absceso perianal, enfermedad inflamatoria, condilomas y/o cáncer anorrectal. Todos los pacientes intervenidos cumplían con los criterios para cirugía ambulatoria del CASR, que incluye sujetos mayores de 15 años, clasificación ASA I o II e índice de masa corporal menor o igual a 30 .

\section{Método de evaluación}

Se realizó una encuesta a cada uno de los pacientes operados. La encuesta incluyó los síntomas más frecuentes de enfermedad hemorroidal: sangrado, dolor, prolapso hemorroidal, prurito e incontinencia fecal.

A los 6 o más meses posoperatorios se consultó de manera retrospectiva, vía telefónica, por la frecuencia de los síntomas en el preoperatorio, al $1^{\text {er }}$ mes y al $6^{\circ}$ mes poscirugía. Los síntomas de sangrado, dolor, prolapso hemorroidal y prurito fueron evaluados cualitativamente según escala de Likert. La incontinencia fecal se evaluó mediante la escala de Jorge-Wexner ${ }^{13}$. Finalmente, se consideraron dos preguntas de satisfacción del procedimiento y sus resultados, las que fueron definidas por las autoras, enfocadas en nuestro centro.

Para la evaluación de complicaciones posoperatorias, se revisó la ficha clínica de cada paciente, incluidos los sujetos que no respondieron la encuesta telefónica.

\section{Técnica quirúrgica}

La técnica de hemorroidectomía efectuada fue convencional, basada en la técnica de Ferguson ${ }^{14}$ modificada. Brevemente, paciente en posición proctológica y bajo anestesia epidural, previa infiltración de cada paquete hemorroidal con $2 \mathrm{mg}$ de bupivacaína. Se realizó disección de paquetes hemorroidales por separado, ligadura del paquete en su base, luego resección con electrobisturí, reparación del defecto mucoso con puntos continuos engarzados de Vicryl ${ }^{\mathbb{R}}$ 3-0. El cierre fue hasta el margen anal procurando hemostasia prolija, dejando la zona de piel abierta. Se extrajo un máximo de 2 paquetes hemorroidales por procedimiento. En caso de existir un tercer paquete, se procedió a ligar con punto en pedículo con Vicryl ${ }^{\circledR}$ 3-0 en X.

\section{Manejo del posoperatorio}

El manejo posoperatorio, si bien fue estandarizado, se ajustó a las condiciones de cada paciente. 
Todos los sujetos operados fueron dados de alta el mismo día de su procedimiento cumpliendo los protocolos de cirugía ambulatoria del CASR. Los controles posoperatorios se realizaron a la semana, al mes, a los 3 y 6 meses, ajustados según la evolución de cada paciente.

\section{Variables y análisis estadístico}

Se analizaron variables demográficas, fecha de cirugía, el grado de hemorroides internos (I a IV) según clasificación de Banov et al. ${ }^{15}$ Se definió como hemorroides mixtos a los grado IV que tenían componente externo.

Los síntomas hemorroidales de sangrado, prurito, dolor y prolapso, tanto pre como posoperatorios se catalogaron en la encuesta como variables nominales de 5 niveles, que para el análisis posterior fueron dicotomizadas de acuerdo al grado de sintomatología. Así, se definió como sintomatología clínicamente significativa cuando el síntoma se presentaba más frecuente que una vez al mes (una vez a la semana o más según la encuesta). El síntoma incontinencia fue evaluado cuantitativamente con la escala de Jorge Wexner.

Para el análisis estadístico se utilizó el programa SPSS 20.0. Se utilizaron números totales, medianas y porcentajes. La descripción de la sintomatología consideró los datos categorizados según la encuesta y un posterior análisis con los datos dicotomizados según las definiciones establecidas. El análisis estadístico de los datos dicotomizados se realizó a través del test de McNemar para variables categóricas pareadas. El síntoma incontinencia fue evaluado mediante la comparación de medianas utilizando el test de Wilcoxon para variables cuantitativas pareadas. Se consideró un valor $\mathrm{p}$ significativo $<0,01$ considerando el ajuste por comparaciones múltiples [valor $\mathrm{p}<(0,05 / 3)]$.

\section{Resultados}

En el período comprendido entre junio de 2015 a enero de 2016 se realizaron un total de 54 hemorroidectomías convencionales electivas en el Servicio de Cirugía Ambulatoria del CASR. Del total de sujetos operados, 43 pacientes $(80 \%)$ respondieron la encuesta, 4 pacientes rechazaron responderla y 7 pacientes no contestaron al llamado telefónico. El grupo de estudio de este trabajo corresponde a los 43 pacientes que respondieron la encuesta.

La mediana de edad fue de 56 años (17-80), 26 pacientes $(60 \%)$ fueron mujeres y $17(40 \%)$ fueron hombres. La mediana de tiempo de evaluación y seguimiento fue de 8 meses (6-12).
Al momento de someterse a cirugía, el 56\% de los pacientes tenía hemorroides grado III y IV. Adicionalmente, el $37 \%$ de los pacientes tenía hemorroides grado IV con componente externo (hemorroides mixtas). La mediana de paquetes hemorroidales por paciente al momento de la cirugía fue de 2 (1-4).

De los 54 pacientes operados entre junio de 2015 a enero de 2016, uno $(1,9 \%)$ presentó complicaciones en el seguimiento al mes posquirúrgico, consistente en un absceso perianal que requirió drenaje quirúrgico.

Considerando las 5 dimensiones de síntomas: sangrado, prurito, dolor, sensación de prolapso hemorroidal e incontinencia fecal, 34 pacientes $(79 \%)$ presentaban 3 o más síntomas preoperatorios considerados clínicamente significativos.

Los resultados descritos se resumen en la Tabla 1.

En la Figura 1 se presenta la evolución de la sintomatología entre el preoperatorio y los 6 o más meses posoperatorios, de acuerdo a los 5 niveles de frecuencia consultados, con los datos obtenidos directamente de la encuesta, sin dicotomizar. La persistencia de síntomas a los 6 o más meses posoperado en cada una de las dimensiones analizadas fluctúa entre 9 a 16\%, con excepción de la sensación de prolapso cuya persistencia alcanza hasta un $28 \%$.

En el análisis estadístico con los síntomas dicotomizados según significancia clínica, se evidenció una disminución significativa (valor $\mathrm{p}<0,01$ ).

Tabla 1. Características demográficas y de sintomatología preoperatoria de los pacientes encuestados

\begin{tabular}{|lc|}
\hline & $\mathrm{n}=43$ \\
Edad (años, mediana, rango) & $56(17-80)$ \\
Sexo (mujeres) & $26(60 \%)$ \\
Grado de hemorroides & \\
$\quad$ I & 0 \\
II & $3(7 \%)$ \\
III & $15(35 \%)$ \\
IV & $9(21 \%)$ \\
Mixtos (IV con componente externo) & $16(37 \%)$ \\
Cantidad de paquetes hemorroidales & $2(1-4)$ \\
Síntomas clínicamente significativos en el preop & \\
1 síntoma & $4(9 \%)$ \\
2 síntomas & $5(12 \%)$ \\
3 o más síntomas & $34(79 \%)$ \\
\hline
\end{tabular}

Abreviaciones: preop: preoperatorio. 


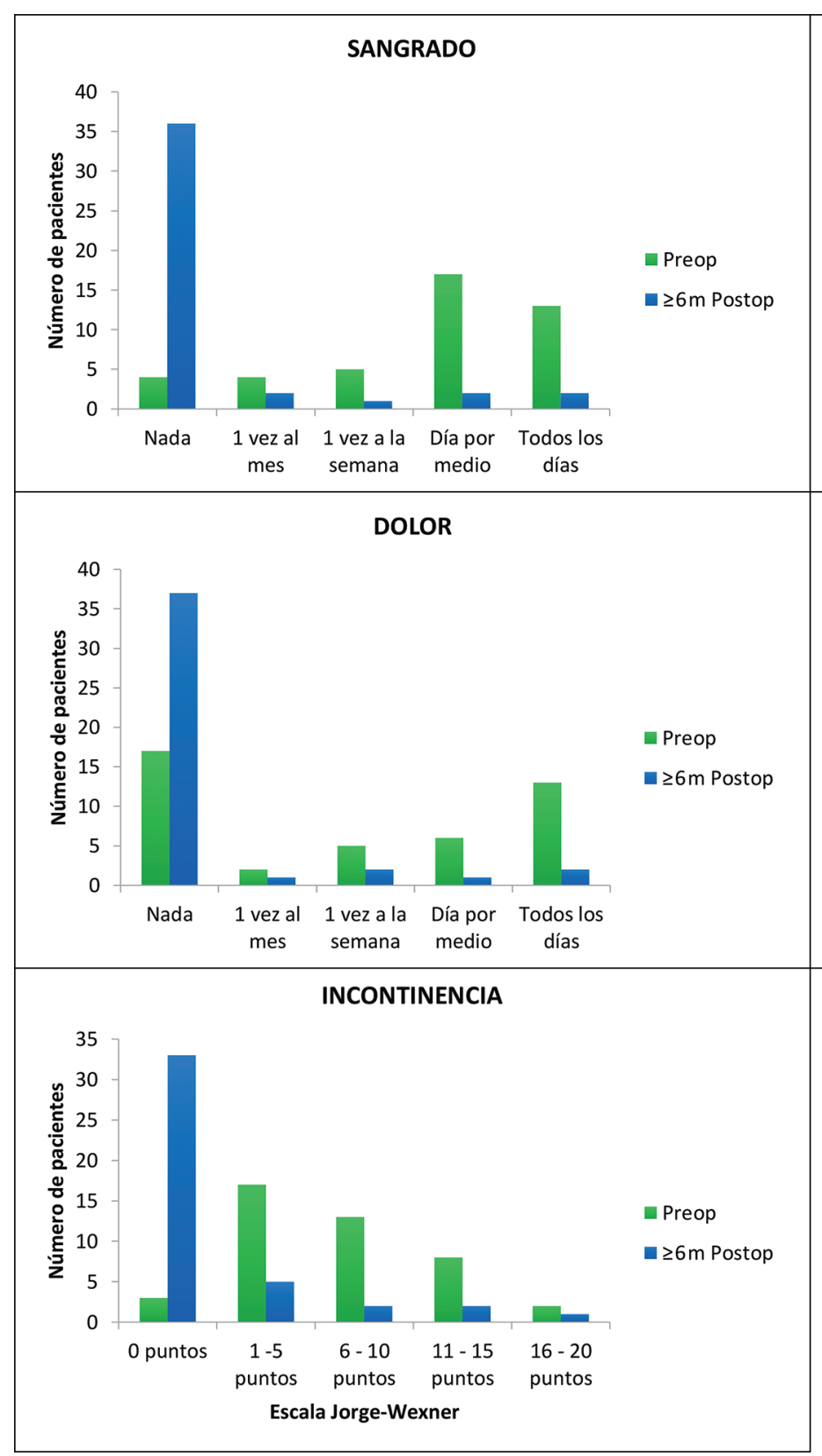

PRURITO

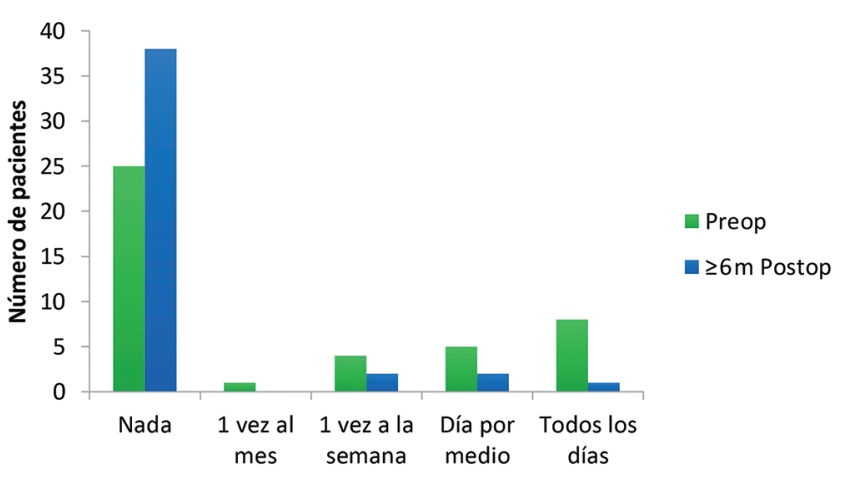

PROLAPSO

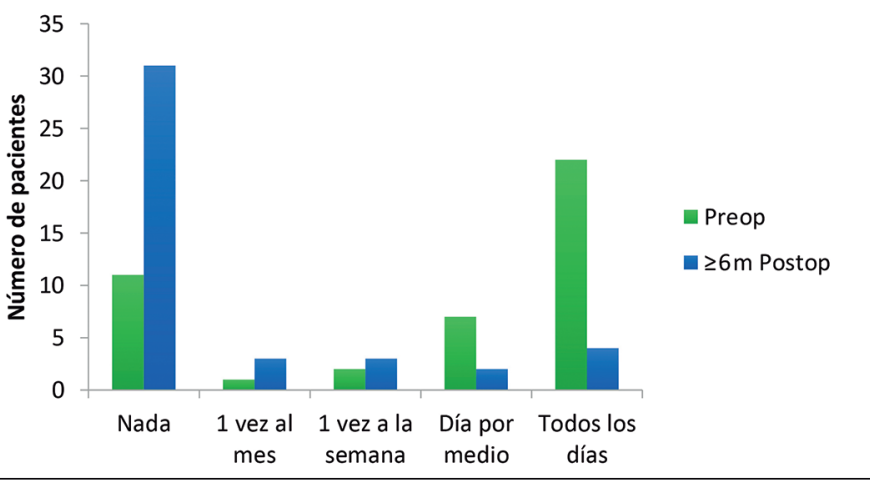

Figura 1. Evolución de los 5 síntomas estudiados entre el preoperatorio y los 6 o más meses posoperatorios. Abreviaciones: Preop: preoperatorio. $\geq 6 \mathrm{~m}$ Posop: 6 o más meses posoperatorios. entre los síntomas evaluados en el preoperatorio comparados con la condición sintomática al mes de operado. La disminución porcentual también es significativa al comparar los síntomas descritos en el prequirúrgico con la condición sintomática a los 6 o más meses posoperatorios. La incontinencia fecal por su parte, presenta una disminución significativa en el análisis de medianas, al comparar la condición prequirúrgica con la sintomatología al mes y a los 6 o más meses de seguimiento. Los porcentajes y medianas se detallan en la Tabla 2.

Con respecto a las preguntas de satisfacción del procedimiento y sus resultados, 42 pacientes respondieron ambas preguntas. Un total de 36 pacientes $(83 \%)$ respondieron en forma afirmativa a la primera interrogante (operarse nuevamente de hemorroides si se estuviese en la misma condición que antes de la cirugía). Frente a la pregunta de recomendación de la cirugía a un tercero, 36 pacientes $(83 \%)$ respondieron en forma afirmativa. 
Tabla 2. Análisis estadístico de los 5 síntomas estudiados, dicotomizados según significancia clínica, entre la condición prequirúrgica y la evaluación al mes y a los 6 o más meses poscirugía

\begin{tabular}{|c|c|c|c|c|c|}
\hline Síntomas & Preoperatorio $^{A}$ & $\begin{array}{c}1^{\mathrm{er}} \text { mes } \\
\text { posoperatorio }^{\mathrm{B}}\end{array}$ & $\begin{array}{l}60 \text { más meses } \\
\text { posoperatorios }^{C}\end{array}$ & Valor $\mathbf{p}^{\mathrm{AB}}$ & Valor $\mathbf{p}^{\mathrm{AC}}$ \\
\hline Sangrado & $91 \%$ & $21 \%$ & $16 \%$ & $<0,01$ & $<0,01$ \\
\hline Dolor & $61 \%$ & $23 \%$ & $12 \%$ & $<0,01$ & $<0,01$ \\
\hline Prolapso & $74 \%$ & $21 \%$ & $28 \%$ & $<0,01$ & $<0,01$ \\
\hline
\end{tabular}

$\mathrm{AB}$ valor $\mathrm{p}$ : valor $\mathrm{p}$ obtenido comparando columnas $\mathrm{A}$ y $\mathrm{B}$ con $\mathrm{McNemar}$ test. $\mathrm{AC}$ valor $\mathrm{p}$ : valor $\mathrm{p}$ obtenido comparando columnas $\mathrm{A}$ y $\mathrm{C}$ con McNemar test. *Síntoma incontinencia: AB y AC analizado con test de Wilcoxon para comparación de medianas.

\section{Discusión}

Una hemorroidectomía ideal es aquella cuya técnica quirúrgica se adecúa al tipo de paciente y su sintomatología, que reseca sólo el grupo hemorroidal afectado, conservando adecuados puentes mucosos para evitar estenosis y manteniendo una buena distención anal posoperatoria ${ }^{7}$. La hemorroidectomía convencional se ha mantenido como una alternativa válida para el tratamiento quirúrgico de la patología hemorroidal a pesar de la emergencia de nuevas técnicas. Como tratamiento a mediano y largo plazo, ha demostrado una menor recidiva con respecto a las otras técnicas quirúrgicas descritas en la literatura ${ }^{10}$. Es uno de los procedimientos más efectivos en pacientes con hemorroides internas grado III y IV ${ }^{16-18}$.

El objetivo de este estudio fue evaluar la evolución de la sintomatología hemorroidal a corto plazo, en los pacientes sometidos a hemorroidectomía clásica, mediante el autorreporte de síntomas a través de encuesta. La creciente conciencia del paciente como centro del sistema de salud y de atención, ha llevado a una mayor preocupación en obtener los resultados reportados por el paciente (PatientReported Outcomes) en las intervenciones clínicas a las cuales ha sido sometido ${ }^{19}$. Su medición y análisis está directamente relacionado con una mejor adherencia a tratamiento y mejores resultados en las intervenciones médicas ${ }^{20}$. En este sentido, nuestro trabajo aporta información valiosa del impacto de la hemorroidectomía clásica desde la perspectiva del paciente.

En nuestra serie, utilizando la técnica convencional, la resolución de síntomas fluctúa entre un $80-90 \%$ a los 6 o más meses posoperatorios, considerando que la mayoría de los pacientes ven resuel- tas sus molestias al mes de operados, y que la sintomatología continúa disminuyendo progresivamente en los meses siguientes. Las complicaciones por su parte fueron de un 1,9\%, las que estaban resueltas a los 6 meses de seguimiento.

El paciente que presentó un absceso perianal, correspondía a un sujeto que respondió la encuesta telefónica posteriormente. En ambas preguntas de satisfacción, respondió en forma afirmativa a someterse nuevamente al procedimiento de hemorroidectomía y recomendar la cirugía a un tercero. De los 11 sujetos que no respondieron la encuesta, ninguno de ellos presentó complicaciones posquirúrgicas descritas al momento de revisar sus fichas clínicas. Con respecto a los pacientes que no recomiendan la cirugía convencional (6 pacientes), uno de ellos corresponde a un encuestado que persistió con incontinencia defecatoria significativa hasta la fecha de seguimiento. En los 5 pacientes restantes, al momento de revisar sus encuestas y sus fichas clínicas, no se constató mayor dolor, recidiva o complicaciones, por lo que pueden existir causas ajenas a la resolución de síntomas, no evaluadas en la encuesta.

Nuestros datos sugieren un alto nivel de satisfacción del paciente con respecto a los resultados de su cirugía, evaluado mediante el uso de preguntas de evaluación subjetiva, alcanzando un $83 \%$ de pacientes que volverían a realizarse el mismo procedimiento o lo recomendarían a un tercero. Estos datos son similares a los obtenidos en seguimientos a mediano y largo plazo en literatura internacional, con porcentajes de satisfacción que varían entre un $70-90 \%$ con el uso de técnica clásica ${ }^{8,12}$. Así, las cifras obtenidas son favorables en contexto de una unidad de cirugía ambulatoria del servicio público de salud de nuestro país. En nuestro estudio, la 
evaluación de la sintomatología, tanto preoperatoria como posoperatoria se realiza de forma retrospectiva, lo cual aumenta el sesgo de reporte y memoria, siendo una clara limitación de este trabajo.

Los futuros análisis de la patología hemorroidal en nuestro país debiesen incorporar la utilización de escalas estandarizadas para la evaluación de sintomatología y calidad de vida, que permitan una comparación objetiva y sistematizada de los distintos resultados obtenidos con el uso de una técnica en particular, seguimiento de pacientes o para la comparación de las nuevas técnicas quirúrgicas emergentes.

En conclusión, la hemorroidectomía convencional es bien evaluada por los pacientes de nuestro centro en relación a una resolución significativa de síntomas de la enfermedad hemorroidal.

\section{Responsabilidades éticas}

Protección de personas y animales. Los autores declaran que para esta investigación no se han realizado experimentos en seres humanos ni en animales.

Confidencialidad de los datos. Los autores declaran que han seguido los protocolos de su centro de trabajo sobre la publicación de datos de pacientes.

Derecho a la privacidad y consentimiento informado. Los autores declaran que en este artículo no aparecen datos de pacientes.

\section{Conflicto de intereses}

Los autores declaran no tener ningún conflicto de intereses.

\section{Anexo 1. Formato de encuesta utilizada para la evaluación de síntomas en pacientes operados de hemorroidectomía}

\section{ENCUESTA PACIENTES OPERADOS DE HEMORROIDECTOMÍA}

Identificación del paciente: Nombre - RUT - Edad - Género - Fecha de cirugía

\section{Evaluación Preoperatoria}

Sangrado:

\begin{tabular}{|c|c|c|c|c|}
\hline Todos los días & Día por medio & Una vez a la semana & Una vez al mes & No tiene \\
\hline \multicolumn{5}{|l|}{ Prurito: } \\
\hline Todos los días & Día por medio & Una vez a la semana & Una vez al mes & No tiene \\
\hline \multicolumn{5}{|l|}{ Dolor: } \\
\hline Todos los días & Día por medio & Una vez a la semana & Una vez al mes & No tiene \\
\hline
\end{tabular}

Prolapso o sensación de aumento de volumen anal en relación a las deposiciones:

\begin{tabular}{|c|c|c|c|c|}
\hline Todos los días & Día por medio & Una vez a la semana & Una vez al mes & No tiene \\
\hline
\end{tabular}

\begin{tabular}{|c|c|c|c|c|c|}
\hline IIPO & Nunca & Rara & $\begin{array}{c}\begin{array}{c}\text { Algunas } \\
\text { veces }\end{array} \\
\text {. }\end{array}$ & Generalmente & Siempre \\
\hline Solidos & 0 & 1 & 2 & 3 & 4 \\
\hline Liquidos & 0 & 1 & 2 & 3 & 4 \\
\hline Gas & 0 & 1 & 2 & 3 & 4 \\
\hline Uso de apósitos & 0 & 1 & 2 & 3 & 4 \\
\hline $\begin{array}{l}\text { Alteración del } \\
\text { estilo de vida }\end{array}$ & 0 & 1 & 2 & 3 & 4 \\
\hline
\end{tabular}

Score Jorge-Wexner: 


\section{Evaluación al mes posoperatorio:}

Sangrado:

\begin{tabular}{|l|l|l|l|l|}
\hline Todos los días & Día por medio & Una vez a la semana & Una vez al mes & No tiene \\
\hline
\end{tabular}

Prurito:

\begin{tabular}{l|l|l|l|l} 
Todos los días & Día por medio & Una vez a la semana & Una vez al mes & No tiene
\end{tabular}

Dolor:

\begin{tabular}{|l|c|c|c|c|}
\hline Todos los días & Día por medio & Una vez a la semana & Una vez al mes & No tiene \\
\hline
\end{tabular}

Prolapso o sensación de aumento de volumen anal en relación a las deposiciones: \begin{tabular}{l|c|c|c} 
Todos los días & Día por medio & Una vez a la semana & Una vez al mes
\end{tabular}

\begin{tabular}{|c|c|c|c|c|c|}
\hline \multicolumn{6}{|c|}{ TABLA 3. SCORE DE INCONTINENCIA DE WEXNER } \\
\hline TIPO & Nunca & Rara & \begin{tabular}{|c|}
$\begin{array}{c}\text { Algunas } \\
\text { veces }\end{array}$ \\
\end{tabular} & Generalmente & Siempre \\
\hline Solidos & 0 & 1 & 2 & 3 & 4 \\
\hline Liquidos & 0 & 1 & 2 & 3 & 4 \\
\hline Gas & 0 & 1 & 2 & 3 & 4 \\
\hline Uso de apósitos & 0 & 1 & 2 & 3 & 4 \\
\hline $\begin{array}{l}\text { Alteración del } \\
\text { estilo de vida }\end{array}$ & 0 & 1 & 2 & 3 & 4 \\
\hline
\end{tabular}

Score Jorge-Wexner:

\section{Evaluación a los 6 o más meses posoperatorios:}

Sangrado:

\begin{tabular}{|l|l|l|c|c|}
\hline Todos los días & Día por medio & Una vez a la semana & Una vez al mes & No tiene \\
\hline
\end{tabular}

Prurito:

\begin{tabular}{|l|l|l|l|l|}
\hline Todos los días & Día por medio & Una vez a la semana & Una vez al mes & No tiene \\
\hline
\end{tabular}

Dolor:

Dolor:
\begin{tabular}{|c|c|c|c|c|}
\hline Todos los días & Día por medio & Una vez a la semana & Una vez al mes & No tiene \\
\hline
\end{tabular}

Prolapso o sensación de aumento de volumen anal en relación a las deposiciones:

\begin{tabular}{l|c|c|c} 
Todos los días & Día por medio & Una vez a la semana & Una vez al mes
\end{tabular}

\begin{tabular}{|c|c|c|c|c|c|}
\hline \multicolumn{6}{|c|}{ TABLA 3. SCORE DE INCONTINENCIA DE WEXNER } \\
\hline IIPO & Nunca & Rara & $\begin{array}{l}\begin{array}{c}\text { Algunas } \\
\text { veces }\end{array} \\
\end{array}$ & Generalmente & Siempre \\
\hline Sólidos & 0 & 1 & 2 & 3 & 4 \\
\hline Liquidos & 0 & 1 & 2 & 3 & 4 \\
\hline Gas & 0 & 1 & 2 & 3 & 4 \\
\hline Uso de apósitos & 0 & 1 & 2 & 3 & 4 \\
\hline $\begin{array}{l}\text { Alteración del } \\
\text { estilo de vida }\end{array}$ & 0 & 1 & 2 & 3 & 4 \\
\hline \multicolumn{6}{|c|}{ 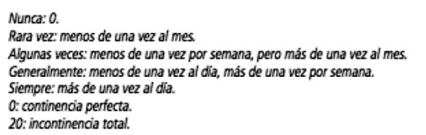 } \\
\hline
\end{tabular}

Score Jorge-Wexner:

\section{Preguntas de satisfacción:}

¿Se operaria Ud. nuevamente de hemorroides si estuviera en la misma condición que antes de operarse? Sí No

¿Recomendaría la cirugía a otras personas con el mismo problema? 


\section{Bibliografía}

1. Sneider EB, Maykel JA. Diagnosis and management of symptomatic hemorrhoids. Surg Clin North Am. 2010;90:17-32.

2. Sardinha TC, Corman ML. Hemorrhoids. Surg Clin North Am. 2002;82:1153-67.

3. Gencosmanoglu R, Sad O, Koç D, Inceoğlu R. Hemorrhoidectomy: open or closed technique? Dis Colon Rectum 2002;45:70-5.

4. Johanson JF, Sonnenberg A. The prevalence of hemorrhoids and chronic constipation. An epidemiologic study. Gastroenterology 1990;98:380.

5. MacRae HM, McLeod RS. Comparison of hemorrhoidal treatments: a meta-analysis. Can J Surg. 1997;40:14-27.

6. Parks AG. The surgical of haemorrhoids. Br J Surg. 1956;43:337-57.

7. Rivadeneira DE, Steele SR, Ternent C, Chalasani S, Buie WD, Rafferty JL. Practice parameters for the management of hemorrhoids (revised 2010). Dis Colon Rectum 2011;54:1059-64.

8. Milone M, Maietta P, Leongito M, Pesce G, Salvatore G, Milone F. Ferguson hemorrhoidectomy: is still the gold standard treatment? Updates Surg. 2012;64:191-4.

9. Riss S, Weiser FA, Schwameis K, Riss T, Mittlböck M, Steiner G, et al. The prevalence of hemorrhoids in adults. Int $\mathrm{J}$ Colorectal Dis. 2012;27:215-20.

10. Simillis $C$, Thoukididou SN, Slesser AA, Rasheed S, Tan E, Tekkis PP. Systematic review and network metaanalysis comparing clinical outcomes and effectiveness of surgical treatments for haemorrhoids. Br J Surg. 2015;102:160318.

11. Watson AJ, Hudson J, Wood J, Kilonzo M, Brown SR, McDonald A, et al. Comparison of stapled haemorrhoidopexy with traditional excisional surgery for haemorrhoidal disease (eTHoS): a pragmatic, multicentre, randomised controlled trial. Lancet 2016;388:2375-85.

12. Guenin MO, Rosenthal R, Kern B, Peterli R, von Flüe M, Ackermann C. Ferguson hemorrhoidectomy: long-term results and patient satisfaction after Ferguson's hemorrhoidectomy. Dis Colon Rectum 2005;48:1523-7.

13. Jorge JM, Wexner SD. Etiology and management of fecal incontinence. Dis
Colon Rectum 1993;36:77-97.

14. Ferguson J, Maizer W, Ganchrow M, Friend W. The Closed Technique of Hemorrhoidectomy. Surgery 1971;70:4804.

15. Banov L Jr, Knoepp LF Jr, Erdman LH, Alia RT. Management of hemorrhoidal disease. J S C Med Assoc. 1985;81:398401.

16. MacRae HM, Mc Leod RS. Comparison of hemorrhoidal treatament modalities: A meta analysis. Dis Colon Rectum 1995;38:687-94.

17. Granet E. Hemorrhoidectomy failures: causes, prevention and management. Dis Colon Rectum 1968;11:45-8.

18. McConnell JC, Khubchandani IT. Long-term follow-up of closed hemorrhoidectomy. Dis Col Rect. 1983;26:797-9.

19. Deshpande PR, Rajan S, Sudeepthi BL, Abdul CP. Patient-reported outcomes: A new era in clinical research. Perspect Clin Res. 2011;2:137-44.

20. 1st Ed. Washington, DC: National Academies Press; 2001. Institute of Medicine, Crossing the Quality Chasm-A New Health System for the $21^{\text {st }}$ Century. 